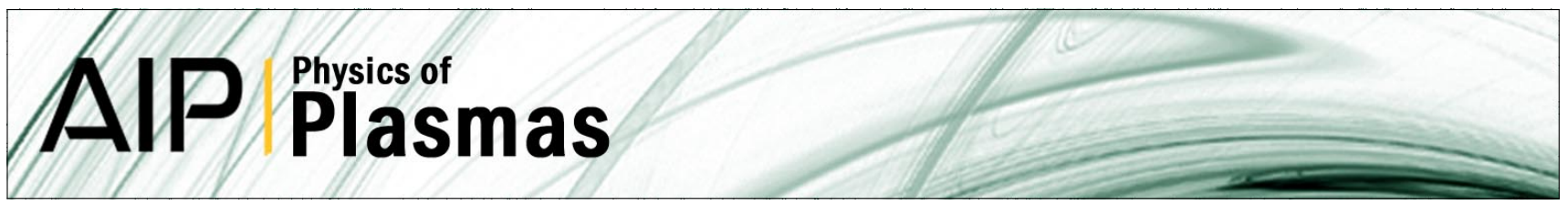

\title{
Lévy walk description of suprathermal ion transport
}

\author{
K. Gustafson and P. Ricci
}

Citation: Phys. Plasmas 19, 032304 (2012); doi: 10.1063/1.3690097

View online: http://dx.doi.org/10.1063/1.3690097

View Table of Contents: http://pop.aip.org/resource/1/PHPAEN/v19/i3

Published by the American Institute of Physics.

\section{Related Articles}

Transport coefficients in strongly coupled plasmas

Phys. Plasmas 19, 030701 (2012)

Surface thermocouples for measurement of pulsed heat flux in the divertor of the Alcator C-Mod tokamak Rev. Sci. Instrum. 83, 033501 (2012)

Ring-shaped velocity distribution functions in energy-dispersed structures formed at the boundaries of a proton stream injected into a transverse magnetic field: Test-kinetic results

Phys. Plasmas 19, 022903 (2012)

Dust charging processes in the nonequilibrium dusty plasma with nonextensive power-law distribution Phys. Plasmas 19, 023704 (2012)

Modification of anisotropic plasma diffusion via auxiliary electrons emitted by a carbon nanotubes-based electron gun in an electron cyclotron resonance ion source

Rev. Sci. Instrum. 83, 02A343 (2012)

\section{Additional information on Phys. Plasmas}

Journal Homepage: http://pop.aip.org/

Journal Information: http://pop.aip.org/about/about_the_journal

Top downloads: http://pop.aip.org/features/most_downloaded

Information for Authors: http://pop.aip.org/authors

\section{ADVERTISEMENT}

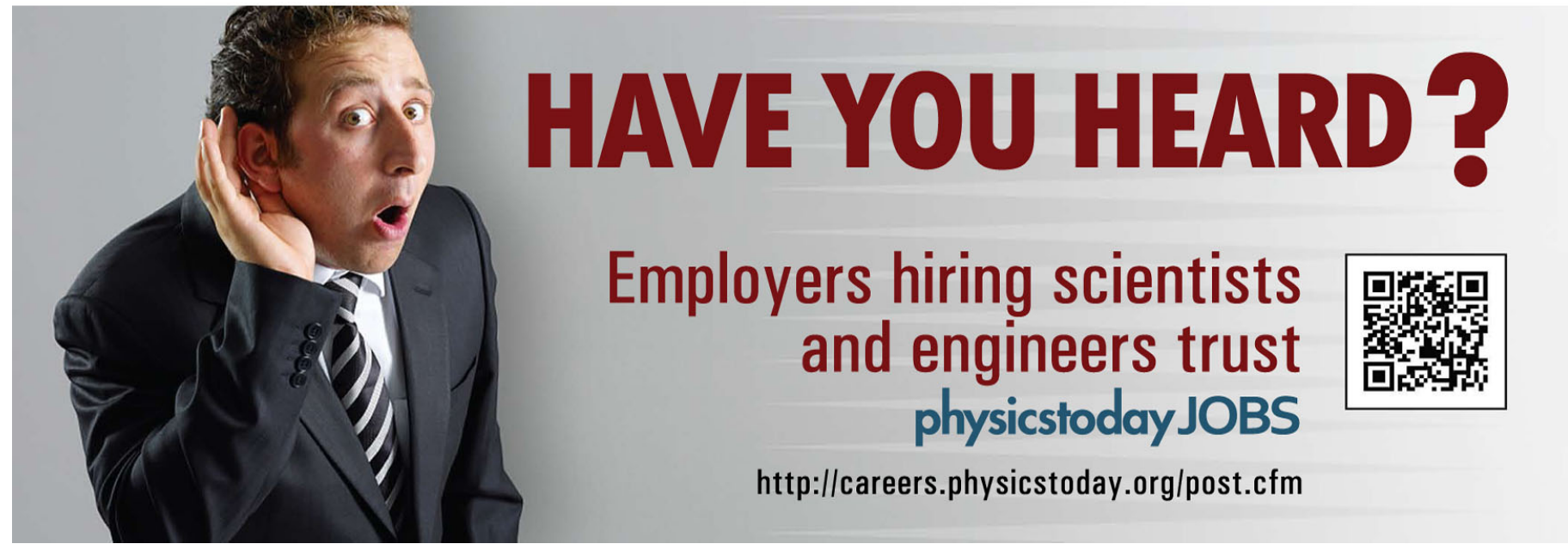




\title{
Lévy walk description of suprathermal ion transport
}

\author{
K. Gustafson ${ }^{\text {a) }}$ and P. Ricci \\ Ecole Polytechnique Fédérale de Lausanne (EPFL), Centre de Recherches en Physique des Plasmas, \\ Association Euratom-Confédération Suisse, CH-1015 Lausanne, Switzerland
}

(Received 23 December 2011; accepted 2 February 2012; published online 7 March 2012)

\begin{abstract}
Transport of suprathermal ions is examined from the Lévy walk perspective in a simple magnetized toroidal plasma. Depending on their energy, these suprathermal ions exhibit superdiffusive, diffusive, or subdiffusive dispersion as a result of the complex interplay between ion drifts related to the magnetic field configuration and interaction of the ions with the plasma turbulence. By implementing a diagnostic that translates the ion trajectories into sequences of steps, we successfully describe their microscale dynamics as a Lévy walk process. Previous analytical predictions that link the microscale Lévy walk parameters to the macroscale suprathermal ion transport are confirmed for all observed regimes of ion dispersion. Additionally, we employ a statistical Lévy walk generator for a direct comparison between transport of Lévy walkers and of suprathermal ions, further validating the Lévy walk description. [http://dx.doi.org/10.1063/1.3690097]
\end{abstract}

\section{INTRODUCTION}

A wide range of plasma systems, ranging from magnetically confined plasmas for fusion to space plasmas, are characterized by the presence of suprathermal ions. They can be generated by turbulent acceleration, ${ }^{1}$ external sources, ${ }^{2-7}$ or, as in the case of fusion devices, nuclear reactions. ${ }^{4,8,9}$ The dynamics of those ions results from the complex interplay between drifts related to the magnetic field configuration and interactions with the plasma turbulence. ${ }^{10}$ Finding an effective way to describe and characterize suprathermal ion dynamics is a key challenge for basic plasma physics and is of significant importance for the success of tokamaks. ${ }^{3-5,8}$

From a macroscale perspective, transport of suprathermal ions is often described with a diffusion equation. On the other hand, a microscale view of the transport process can provide insights, especially when the transport process does not obey the diffusion equation. One particularly transparent and easily generalizable microscale approach to describe the spreading of a population of particles, such as an ensemble of suprathermal ions, is the continuous time random walk. ${ }^{11}$ This approach consists of following trajectories of particles, the random walkers, through a sequence of steps. If $\psi(\Delta \mathbf{r}, \Delta t)$ is the probability distribution function (PDF) for a random walker to make a step of length $\Delta \mathbf{r}$ that takes a time $\Delta t$, then the spatial distribution of particles at time $t$ and position $\mathbf{r}$ satisfies the integral equation,

$$
\rho(\mathbf{r}, t)=\int_{0}^{t} d \tau \int d \mathbf{r}^{\prime} \rho\left(\mathbf{r}^{\prime}, \tau\right) \psi\left(\mathbf{r}-\mathbf{r}^{\prime}, t-\tau\right) .
$$

In the case that the step-size PDF is separable, $\psi(\Delta \mathbf{r}, \Delta t)=$ $\lambda(\Delta \mathbf{r}) \phi(\Delta t)$, where $\lambda(\Delta \mathbf{r})$ is a normal distribution, and $\phi(\Delta t)$ is a Poisson distribution, the diffusion equation for $\rho(\mathbf{r}, t)$,

$$
\partial_{t} \rho(\mathbf{r}, t)=D \nabla^{2} \rho(\mathbf{r}, t)
$$

\footnotetext{
${ }^{a)}$ Electronic mail: kyle.gustafson@epfl.ch.
}

can be deduced from Eq. (1) using Fourier-Laplace transform methods. ${ }^{12}$ In this case, the variance $\sigma^{2}(t)=\int r^{2} \rho(\mathbf{r}, t)$ $d \mathbf{r}$ grows linearly with time, $\sigma^{2}(t)=D t$, as expected for diffusive behavior.

In nondiffusive transport, $\sigma^{2}(t) \propto t^{\gamma}$, where $\gamma \neq 1$. If $\gamma>1$, the transport is called superdiffusive; if $\gamma<1$, the transport is called subdiffusive. Nondiffusive processes have great importance in nature. ${ }^{13}$ In Klafter et al., ${ }^{14}$ it is argued that nondiffusive processes, ranging from subdiffusive to superdiffusive, can result from a form of $\psi$ that couples $\Delta t$ and $\Delta \mathbf{r}$. In one dimension, a general coupling function is

$$
\psi(\Delta r, \Delta t) \propto \Delta r^{-\mu} \delta\left(\Delta t-\alpha \Delta r^{1 / \nu}\right)
$$

where $\mu, \alpha$, and $\nu$ are positive real numbers. Particles subject to steps whose distribution is described by Eq. (3) are said to follow a Lévy walk. In Ref. 14, analytic estimates are provided that relate $\gamma$ to the values of $\mu$ and $\nu$, thus connecting microscale transport properties to the macroscale behavior.

Analogously to a Lévy flight, ${ }^{15}$ Lévy walks allow steps of arbitrary length, according to a power law PDF. However, contrary to the Lévy flight, Lévy walks penalize long steps, since they require more time. Lévy walks have been used to describe transport phenomena in a wide range of systems, from neutral fluids ${ }^{16}$ to the motion of magnetic bright points in the Sun. ${ }^{17}$ In the context of plasma physics, it has been pointed out that superballistic transport observed in a static reconnecting current-sheet is in agreement with the Lévy walk prediction. $^{18}$

In the present paper, we perform an analysis of the turbulent transport of suprathermal ions within the Lévy walk framework. In particular, we consider suprathermal ions in the simple magnetized torus (SMT) configuration, ${ }^{19-24}$ where a vertical magnetic field, $B_{z}$, superimposed with a toroidal magnetic field, $B_{\phi}$, creates helicoidal field lines terminating on the toroidal vessel. This configuration is of interest to the plasma turbulence and fusion communities, as it offers a relatively simple and well-diagnosed test bed in which to study 
the basic physics of plasma turbulence and the associated transport of heat and particles.

The SMT incorporates, in a simplified form, the fundamental elements determining suprathermal ion dynamics in more general contexts. These elements include the $\mathbf{E} \times \mathbf{B}$ drift and polarization drift caused by electrostatic turbulence, Larmor gyration with gyroradius near the scale of the turbulent fluctuations, and drifts related to the shape of the magnetic field. From a theoretical point of view, the relative simplicity of the SMT configuration allows a complete characterization of turbulence through global simulations validated against experimental data. ${ }^{25,26}$ The results of those unique simulations, which evolve the plasma dynamics resulting from the interplay between the plasma source, losses at the vessel, and turbulence, with no separation between equilibrium and fluctuating quantities, provide an ideal setting for integrating suprathermal ion trajectories.

Indeed, inspired by the suprathermal ion experiment ${ }^{27,28}$ installed in the SMT TORPEX (Refs. 29 and 30), we have conducted a theoretical characterization of suprathermal ion trajectories. These results, presented in Refs. 10 and 31, show a surprisingly wide range of $\gamma$ values covering all regimes described by the Lévy walk model, depending on the injection energy and the amplitude of the turbulence. Simulation results have been favorably compared with initial experimental results. ${ }^{28}$ Therefore, the SMT provides an excellent context for a study of the validity of the Levy walk description of suprathermal ion transport.

In the present paper, by analyzing suprathermal ion trajectories in the SMT simulated fields and also using a statistical Lévy walk generator, we show that the Lévy walk model is a transparent and flexible framework to describe suprathermal ion transport, capable of explaining the appearance of subdiffusive, diffusive, and superdiffusive transport regimes. We discuss how the microscale parameters of the Lévy walk model, $\mu$ and $\nu$, can be measured, and we analyze their relationship to the macroscale parameter $\gamma$. We also evaluate the agreement of our numerical findings with the theoretical predictions discussed in Ref. 14.

The content of this paper is organized into the following five additional sections. In Sec. II we describe the properties of suprathermal ion trajectories in the SMT, summarizing the results of Refs. 10 and 31. Section III presents a Lévywalk-based analysis of suprathermal ion transport in an SMT. The implementation of a Lévy walk generator and the comparison of its results with the suprathermal ion dispersion observed in the SMT are shown in Sec. IV. Finally, we summarize our findings in Sec. V.

\section{SUPRATHERMAL ION DYNAMICS IN THE SMT}

For concreteness, in our work we refer to the parameters of the TORPEX device, ${ }^{29,30}$ an SMT characterized by $B_{v} \ll B_{\phi}, \beta \ll 1$, and $T_{i} \ll T_{e}$. In TORPEX, plasma is generated on the high-field side of the torus by microwave absorption at the electron-cyclotron and upper-hybrid resonances. This localized plasma source leads to a pressure gradient that, together with the curvature of the magnetic field, triggers a number of instabilities. These instabilities lead to different turbulence regimes that have been characterized both experimentally and theoretically in TORPEX. ${ }^{32-34}$

Here, we focus on the ideal interchange instability, which is dominant for a sufficiently high $B_{v}$ and low plasma resistivity. ${ }^{33}$ In this regime, $k_{\|}=0$ and the wavelength of the dominant mode along $z$ [the direction perpendicular to both $B$ and the radial direction, which is tilted by an angle $\theta=$ $\tan ^{-1}\left(B_{v} / B_{\phi}\right) \ll 1$ with respect to the vertical direction], is given by the return length of the field line on the poloidal plane, i.e., $\lambda_{z}=\Delta$, where $\Delta=2 \pi R_{0} B_{v} / B_{\phi}$, with $R_{0}$ being the SMT major radius. Since $k_{\|}=0$, the study of plasma turbulence in this regime can be approached by performing twodimensional simulations in the plane perpendicular to the magnetic field. These simulations, based on the drift-reduced Braginskii model, ${ }^{35}$ evolve the plasma density, the electron temperature, and electrostatic potential as a function of time, and have been validated against experimental data. ${ }^{25,26}$

In TORPEX, a suprathermal ion beam is provided by a miniaturized lithium $6+$ ion source. In order to simulate its spatiotemporal behavior, we integrate the non-relativistic ion equation of motion with the Lorentz force considering the SMT magnetic configuration and the electric field provided by the two-dimensional simulations described in Ref. 32 . Injection conditions are chosen to mimic the suprathermal ion source, focusing on the parallel injection, such that the axis of the injection cone is directed along a field line.

Throughout the present paper we focus on ion transport in the radial direction. As discussed in detail in Refs. 10 and 31 , the dynamical behavior of the suprathermal ions can be described as the interplay between radial $\mathbf{E} \times \mathbf{B}$ turbulent velocities, gyroaveraging due to the Larmor motion, and drift-averaging due to curvature and $\nabla B$ drifts. After an initial phase, where the ions display a ballistic spreading, the complex interaction between these elements generally causes nondiffusive transport of the suprathermal ions, such that the variance of ion radial displacements, $\sigma^{2}=\left\langle\delta R^{2}\right\rangle$, grows as $\sigma^{2}(t) \sim t^{\gamma}$. We find that $\gamma$ is approximately constant until the ions have spread radially enough to sample regions where turbulence properties are significantly different than at the injection position. We denote as "interaction regime" the quasi-steady state phase during which $\gamma$ is almost constant, bracketed between the initial ballistic regime and the time at which boundary effects or the radial dependence of turbulence parameters becomes important, leading to a change in $\gamma$. The interaction regime is most interesting for investigations of turbulent transport, and is the focus our attention. Our simulations show that $\gamma$ depends on the relative amplitude of the turbulent fluctuations and the suprathermal ion injection energy normalized to the plasma temperature, such that, surprisingly, $\gamma$ varies continuously from superdiffusive to very small subdiffusive values. ${ }^{10,31}$

Here, we present three representative examples showing three types of dispersion: subdiffusion at $\mathcal{E}=250$, diffusion at $\mathcal{E}=25$, and superdiffusion at $\mathcal{E}=5$, where $\mathcal{E} \equiv m v_{0}^{2} /$ $\left(2 T_{e}\right)$ is the initial ion energy normalized to the plasma electron temperature, $T_{e}$. For these cases, the turbulence amplitude in the SMT is such that $\xi=0.8$, where $\xi \equiv e \tilde{\phi} / T_{e}$ and $\tilde{\phi}$ is the root mean square fluctuation amplitude of the electrostatic potential. In Fig. 1, we show these three cases by 


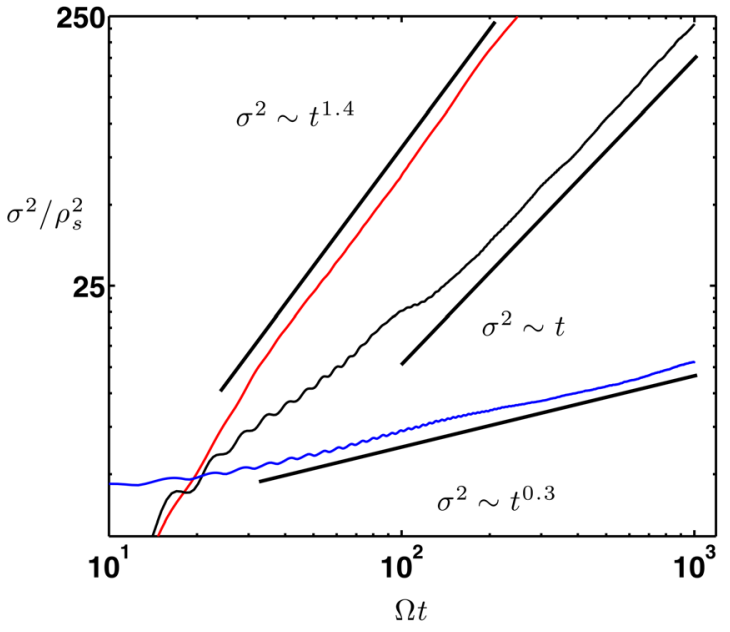

FIG. 1. (Color online) Radial $\sigma^{2}$ for turbulent suprathermal ion transport for three different injection energies $[\mathcal{E}=5$ (red, topmost curve), $\mathcal{E}=25$ (black, middle curve), and $\mathcal{E}=250$ (blue, lower curve)] showing representative examples of superdiffusion, subdiffusion, and diffusion, respectively. We express $\sigma$ in terms of $\rho_{s}$, the ion sound radius for the SMT plasma, and $t$ in terms of $\Omega$, the suprathermal ion Larmor frequency.

plotting $\sigma^{2}$ as a function of time. We determine the value of $\gamma$ by best-fitting the growth of $\sigma^{2}$ during the interaction regime with a power law growth curve. The values of $\gamma$ for the three different cases are summarized in Table I. Error in the fitted values is of the order \pm 0.1 . We follow $10^{4}$ ion trajectories to satisfy statistical convergence with respect to the number of ions. As an aside, we note that periodic oscillations in $\sigma^{2}(t)$ are caused by the ion gyromotion.

The wide variation of $\gamma$ from subdiffusion to superdiffusion makes it very interesting to interpret the suprathermal ion behavior within the Lévy walk framework. In Sec. III, we present how we determine the key elements necessary to describe the ion transport as a Lévy walk process.

\section{ION DYNAMICS DESCRIPTION WITHIN THE LÉVY WALK FRAMEWORK}

The primary goal in this section is to interpret the results of suprathermal ion turbulent transport within the Lévy walk framework. The assumption of coupled step sizes, $\Delta r$, and step durations, $\Delta t$, as given in Eq. (3), enables a determination of the value of $\gamma$ as a function of the two parameters $\nu$ and $\mu .{ }^{14}$ Therefore, our Lévy walk analysis of SMT suprathermal ion transport is reduced to finding the values of these two parameters. In particular $\mu$ can be found from the shape of the step-size PDF, $P(\Delta r)=\int \psi d \Delta t$, while $\nu$ comes from the relation between $\Delta t$ and $\Delta r$.

TABLE I. Measured power law exponents for $\sigma^{2}(t), P(\Delta r)$, and $\Delta r=\Delta r$ $(\Delta t)$. Subscripts indicate either suprathermal ions in the SMT, the prediction of Ref. 14 (KBS), random direction Lévy walk trajectories (RT), and alternating direction Lévy walks (AT).

\begin{tabular}{lcccccc}
\hline \hline & $\gamma_{S M T}$ & $\mu_{S M T}$ & $\nu_{S M T}$ & $\gamma_{K B S}$ & $\gamma_{R T}$ & $\gamma_{A T}$ \\
\hline Superdiffusive & 1.4 & 2.5 & 0.8 & 1.4 & 1.3 & 1.4 \\
Diffusive & 1.0 & 3.5 & 0.9 & 1.0 & 1.0 & 1.0 \\
Subdiffusive & 0.3 & 3.5 & 0.15 & 0.4 & 0.4 & 0.5 \\
\hline \hline
\end{tabular}

It is not obvious how to define a step in a continuous velocity field, in contrast to discrete random walks studied elsewhere. ${ }^{36}$ We have taken the following approach, which is similar to the techniques described in Refs. 18, 37 and 38. First, we gyroaverage the ion trajectories, since we are fundamentally interested in the gyrocenter displacements. Second, we determine the reversal time-points for the radial component of the velocity. Finally, we compute the step size, $\Delta r$, as the radial distance covered by a particle in the time lapse bracketed by two sign reversals of velocity. We note that, in contrast to Lévy flights, we do not distinguish between trapping and flight events. Instead, we identify each trajectory as a sequence of steps of certain lengths, which each take a certain amount of time to be performed.

In Fig. 2, we present the step-size PDF, $P(\Delta r)$, for three representative ensembles of suprathermal ions. Clearly, these distributions are not strictly power laws in the range of step sizes considered. However, we can reasonably focus our attention on the decay of the tails, since they contain the largest displacements, which therefore influence the variation of $\sigma^{2}(t)$ most strongly. In particular, for a fit of the PDF tails with a power law such that $P(\Delta r) \sim \Delta r^{-\mu}$, we choose the range of step sizes containing $50 \%$ of the total distance traveled by suprathermal ions during the interaction regime and corresponds to $\lesssim 20 \%$ of the total number of steps.

The values of $\mu$ found for the superdiffusive, diffusive, and subdiffusive turbulent suprathermal ion transport processes are summarized in Table I. We first note that the value of $\mu$ for the superdiffusive case is smaller with respect to the subdiffusive and diffusive cases. This accounts for the presence of larger steps, which intuitively can lead to a faster dispersion. On the other hand, the diffusive and subdiffusive cases reveal approximately the same $\mu$.

We remark that the $\mu$ values found herein are larger by a factor of 3 compared to those reported by Zimbardo et al. in the analysis of tearing-mode fluctuations. ${ }^{18}$ In that case, the electric field causes significant ion acceleration and very long flights consistent with small $\mu$. On the other hand, our values of $\mu$ are closer to the ones for tracers in solar turbulence ${ }^{17}$ and

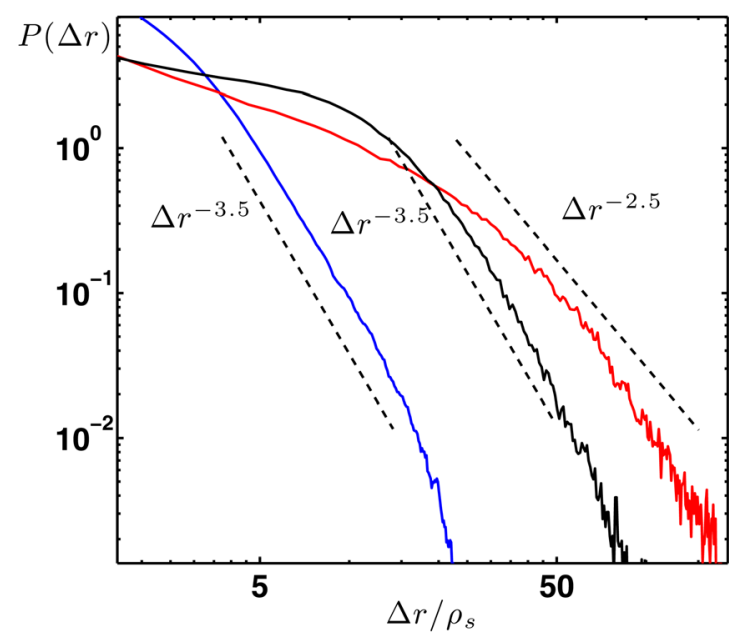

FIG. 2. (Color online) Probability distribution function of step size, $P(\Delta r)$, for suprathermal ions in the SMT with fitted power law tails for the $\mathcal{E}=5$ (red, rightmost curve), $\mathcal{E}=25$ (black, middle curve), and $\mathcal{E}=250$ (blue, leftmost curve). 

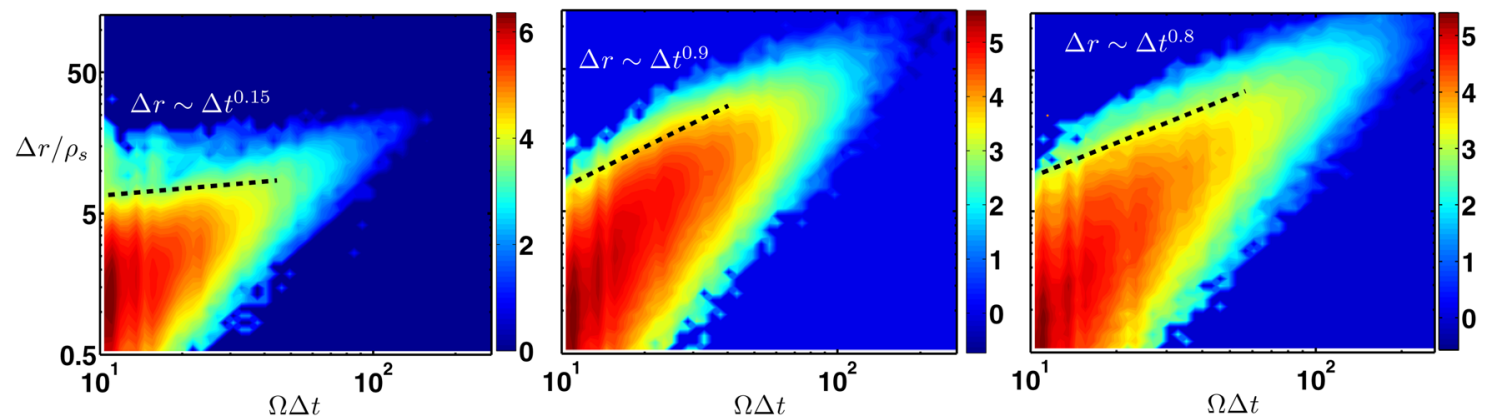

FIG. 3. (Color online) Correlation between $\Delta r$ and $\Delta t$ in log-log scale, with log-scale contours of probability density for steps deduced from suprathermal ion simulations in the SMT. Three cases are shown in order of decreasing energy, from left to right: $\mathcal{E}=250, \mathcal{E}=25$, and $\mathcal{E}=5$. Best fitting power laws for the most relevant parts of the spectra are shown.

in fluid turbulence. ${ }^{16}$ This is consistent with the presence of fully-developed turbulence, without constant acceleration, common to these three contexts.

We now determine the values of $\nu$, the coupling constant. In Fig. 3, we plot the length of each step against its duration for the three cases of interest: superdiffusive, diffusive, and subdiffusive. It is clear that the relation between $\Delta r$ and $\Delta t$ is not given by the simple Lévy walk prediction, $\Delta r=\Delta t^{\nu}$. There is a relatively large uncertainty in determining a value of $\nu$ to fit the relation between $\Delta r$ and $\Delta t$. Therefore, similar to what has been done for the $\mu$ exponent, we focus on the steps that are most responsible for the growth of $\sigma^{2}$, i.e., the steps with the largest $\Delta r$. This is done in Fig. 3, where we have evaluated the value of $\nu$ by fitting the relation between $\Delta t$ and the steps with the largest $\Delta r$ corresponding to each $\Delta t$. We also note that the range of possible $\nu$ values narrows down for the longest step sizes.

In Table I, we summarize the values of $\nu$ from suprathermal ion transport simulations in the SMT for our three representative values of $\mathcal{E}$. Between the superdiffusive and diffusive case, $\nu$ is approximately the same. However, a larger value of $\mu$ alone can reduce $\gamma$ from a superdiffusive value to $\gamma \simeq 1$. Most remarkably, the value of $\nu$ is reduced in the subdiffusive case with respect to the diffusive case, which reflects a larger degree of penalization of long steps. This can intuitively explain, from the Lévy walk perspective, the presence of subdiffusive transport at high $\mathcal{E}$.

In comparison to previous Lévy walk analyses, we note that our superdiffusive and diffusive values of $\nu$ are smaller by a factor of two compared with Ref. 18. This is sensible, since that study of magnetic turbulence in Ref. 18 used a constant, unidirectional accelerating electric field, leading to larger $\Delta r$ for a given $\Delta t$. For the diffusion and superdiffusion reported for the fluid model in Fogleman, ${ }^{16} \nu=1$ by assumption, which is similar to our values for diffusive and superdiffusive $\gamma$. This is smaller than the Richardson value, $\nu=3 / 2$, reported by Lawrence ${ }^{17}$ for solar magnetic bright points. Notably, our subdiffusive $\nu \simeq 0.15$ is smaller than any of the values we found in the literature, consistent with the absence of subdiffusive cases in these other studies.

We now compare our results with the analytical predictions of the dependence of $\gamma$ on $\nu$ and $\mu$ presented in Ref. 14 . For the superdiffusive case, with $\nu \simeq 0.8$ and $\mu \simeq 2.5$, Ref. 14 predicts $\gamma=2-\mu^{\prime} \nu+2 \nu \simeq 1.4$, since $\nu \mu^{\prime}>2$ and $\nu \mu^{\prime}-2 \nu<1$, where $\mu^{\prime}=\mu-1+1 / \nu$, as described in
Ref. 39. Our simulation shows $\gamma \simeq 1.4$. Therefore, the analytical prediction succeeds in predicting the superdiffusive character of the transport with remarkable accuracy. Agreement between the analytical theory and our results is also observed in the diffusive case. In fact, for $\mu=3.5$ and $\nu=0.9$, the analytical prediction confirms the diffusive character of suprathermal ion dispersion. Finally, in the subdiffusive case, where $\mu=3.5$ and $\nu=0.15$, the analytic theory is also confirmed. In fact, for $1<\nu \mu^{\prime}<2$ and $\nu \mu^{\prime}-2>1$, Ref. 14 predicts $\gamma=\nu \mu^{\prime}-1 \simeq 0.4$, which compares very well with the simulated value of $\gamma \simeq 0.3$. Thus, all three examples of suprathermal ion transport match predictions of the Lévy walk theory (see Table I).

\section{LÉVY WALK GENERATOR}

We have implemented a Lévy walk generator, similar to the one reported in Ref. 39, to accomplish two goals. First, we verify that our diagnostic for identifying discrete steps in the continuous process of turbulent transport is able to return reasonably accurate values of $\mu$ and $\nu$. Secondly, our random walk generator allows us to confirm that the fundamental elements of the suprathermal ion transport process are contained in the two main parameters of the Lévy walk, $\nu$ and $\mu$.

In the Lévy walk generator, we follow a statistically converged number of random walkers, drawing their step sizes as pseudo-random numbers from a power law distribution. More precisely, we consider a Pareto PDF of step sizes

$$
P(\Delta r)=\frac{1}{\zeta}\left[\frac{\Delta r}{\zeta(\mu-1)}\right]^{-\mu}
$$

with a cutoff at $\Delta r=\zeta(\mu-1)$, and $\zeta>0$. Steps are assigned with random directions. The duration of each step is given according to the Lévy walk assumption, i.e., $\Delta t=\Delta r^{1 / \nu}$, setting $\alpha=1$. We note no dependence of our results on the cutoff value.

In principle, our diagnostic might not report a correct value of $\nu$ and $\mu$. The diagnostic is based on identifying steps as occurring between velocity reversals, which generally do not occur at every step. This causes the diagnostic to combine several individual steps into a single longer step. However, we find that $\mu$ and $\nu$ can, in general, be measured accurately. As shown in Fig. 4, the tails of $P(\Delta r)$ are in good 


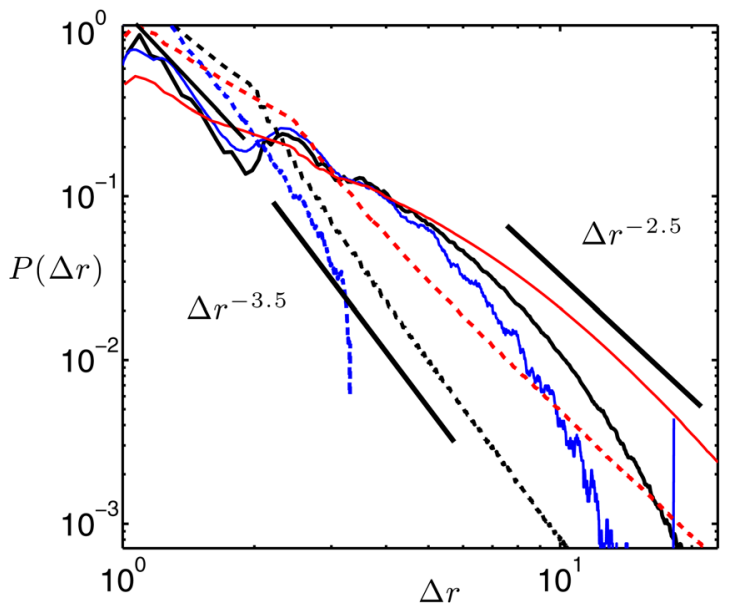

FIG. 4. (Color online) Probability distribution function of step size, $P(\Delta r)$, from the Lévy walk generator for three cases, superdiffusive (red, rightmost curves), diffusive (black, middle curves), and subdiffusive (blue, leftmost curves). The set of solid curves is for randomly alternating step directions, while the set of dashed curves is from the random walk with displacement reversals at every step. Units are arbitrary.

agreement with the expected $\mu$ values in all cases. Also, the sharp coupling between $\Delta r$ and $\Delta t$ dictated by the generator is reproduced in the center of a peaked correlation for diffusive and superdiffusive cases, as Fig. 5 shows. The problematic exception is the subdiffusive case, which shows the larger steps deviating from the coupling of the Lévy walk. These larger steps result from combining a sequence of steps, all following the same direction.

In order to prove that trajectories with randomly alternating directions cause the diagnostic to combine several small steps into a single larger step, thus affecting the results, we have also implemented trajectories with alternating directions, such that the sign of the displacement reverses at each step. This means that no two steps will be combined into a longer step by our diagnostic. As shown in Figs. 4 and 5, in

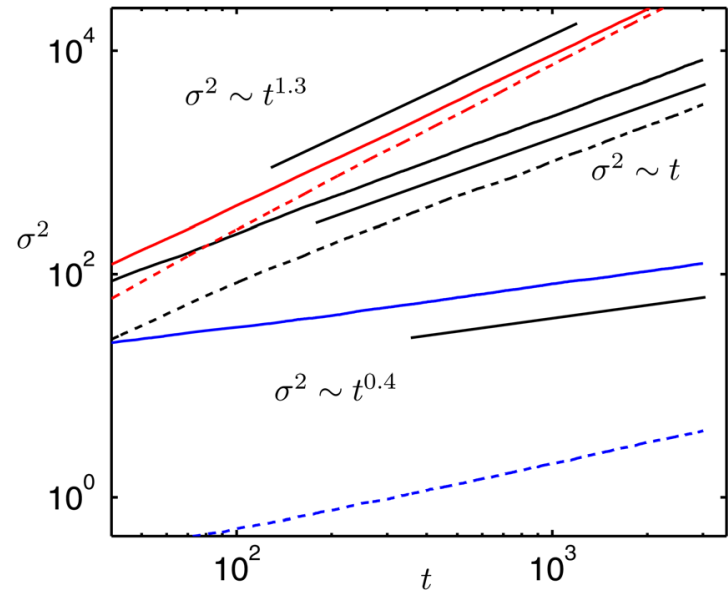

FIG. 6. (Color online) Variance of displacements from the Lévy walk generator, showing converged values of $\gamma(t)$ for superdiffusive (red, topmost curves), diffusive (black, middle curves), and subdiffusive (blue, lower curves) cases chosen to model the turbulence data. The set of solid curves is for randomly alternating step directions, while the set of dashed curves is from random walks with displacement reversals at every step. Units are arbitrary.

this case, the $\mu$ and $\nu$ values are measured with higher certainty than for randomly directed trajectories. We also note that the alternating-sign random walks provide values of $\gamma$ that are in close agreement with the random-sign version, as shown in Fig. 6, where we plot $\sigma^{2}(t)$, and Table I.

The Lévy walk generator also confirms that the transport processes specified by the values of $\nu$ and $\mu$ measured in Sec. III provide approximately the same $\gamma$ values computed in Sec. III, as shown in Fig. 6 and Table I. One can observe that the transport regime is well-identified in all three cases, with values of $\gamma$ that are in close agreement with respect to the ones found from the SMT simulations and the analytical theory.

The results of the Lévy walk generator permit two statements. First, the algorithm introduced for detecting steps
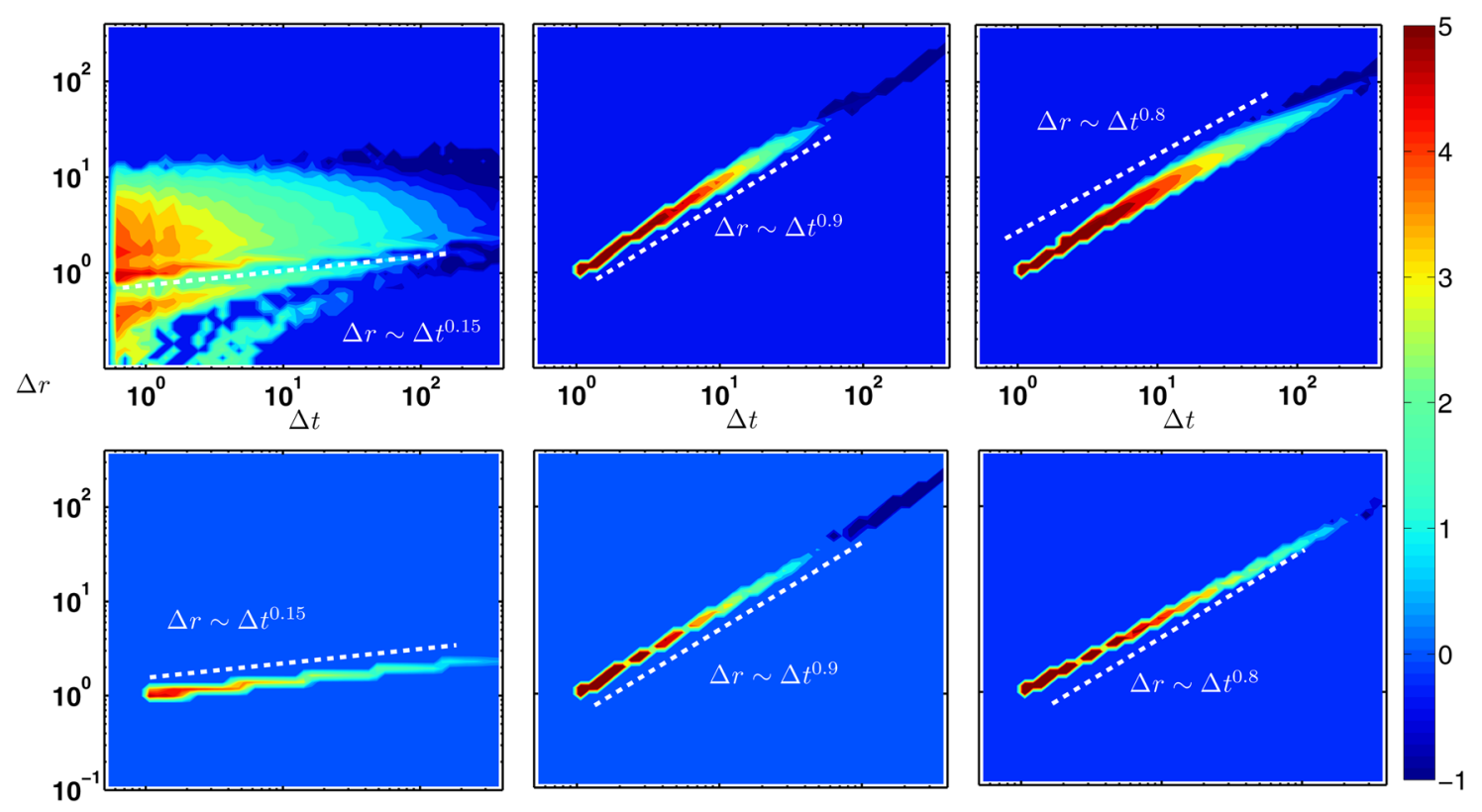

FIG. 5. (Color online) Correlation between $\Delta r$ and $\Delta t$ in log-log scale, with log-scale contours of probability density for steps in the Lévy walk generator. The bottom row is from randomly alternating step directions, while the top row is from random walks with displacement reversals at every step. Units are arbitrary. 
provides measurements of $\nu$ and $\mu$ within a reasonable accuracy, the only caveat being for the measurement of $\nu$ in the subdiffusive case. Second, it is possible to explain the value of $\gamma$ in the superdiffusive, diffusive, and subdiffusive cases within the Lévy walk formalism. Quantitative agreement is achieved for each regime between the results of the Lévy walk generator, the measurements from SMT turbulence and the analytical estimates for Lévy walks given by Ref. 14 .

\section{CONCLUSION}

In this paper, we have examined the transport of suprathermal ions in the SMT configuration within the framework of the Lévy walk description detailed by Ref. 14. We have considered suprathermal ions as tracer particles, and thus have integrated the equation of motion with the Lorentz force in simulated ideal interchange turbulence. These simulations have been validated against experimental data. ${ }^{25,26,28}$ Suprathermal ion transport in the SMT depends on the interplay of $\mathbf{E} \times \mathbf{B}$ turbulent velocity, gyroaveraging, and drift-averaging effects, which causes ions to spread radially in time such that $\sigma^{2} \sim t^{\gamma}$, with $\gamma$ ranging from subdiffusive to superdiffusive values depending on the ion energy and turbulence fluctuation amplitude. This range of transport regimes makes the SMT configuration a useful proving ground for the Lévy walk framework to interpret suprathermal ion dynamics. In contrast to Lévy flights, Lévy walks couple the step size and step duration, thus penalizing longer steps. This allows exploration of transport regimes from subdiffusive to superdiffusive. While other authors have used Lévy walks in fluids ${ }^{16}$ and in plasma turbulence, ${ }^{18}$ we believe that our work constitutes the first application of the Lévy walk to the complete set of applicable transport regimes while using a validated numerical model of suprathermal ions in plasma turbulence.

Our findings reveal that knowledge of the $\nu$ and $\mu$ exponents is sufficient to characterize suprathermal ion spreading. In particular, through a Lévy walk generator, we have shown that a statistical random walk using the $\nu$ and $\mu$ exponents measured from the SMT suprathermal ion spreading simulations provides a value of $\gamma$ that is in reasonably close agreement with the turbulence simulation. Regarding comparison with the analytical theory presented in Ref. 14, we notice that for all cases studied, we observe good agreement between simulation results and analytical theory.

Therefore, the SMT results suggest that, within the Lévy walk framework using $\nu$ and $\mu$ to describe the microscale properties of the transport, it is possible to accurately determine the macroscale transport regime and the value of $\gamma$. Further work focused on predicting the values of $\nu$ and $\mu$ from turbulence properties could further advance the Lévy walk as a useful and general approach to the description and characterization of suprathermal ion transport.

We have focused our attention on suprathermal ion dynamics in the SMT. Although this configuration is relatively simple, it contains all the basic elements for suprathermal ion dynamics in a number of physical situations. In fact, we expect that the results presented herein are insightful for describing suprathermal ion dynamics in other basic plasma physics configurations such as the Large Plasma Device
(LAPD) device, where superdiffusive and subdiffusive ion transport have recently been measured, ${ }^{40,41}$ and in magnetically confined plasmas, where it may be possible to model the interaction of energetic particles with Alfvénic turbulence using the Lévy walk approach. Such considerations may also be relevant for solar wind turbulence, as well as cosmic rays in interstellar magnetic fields.

Finally, we remark that our theoretical study has been inspired by the suprathermal ion experiment in TORPEX. Although this experiment will not easily yield the microscale parameters necessary to determine $\nu$ and $\mu$, it will be able to provide values of $\gamma$ for the suprathermal ion transport, thus confirming or refuting the presence of the different transport regimes consistent with the Lévy walk.

\section{ACKNOWLEDGMENTS}

This work was supported by NSF-IRFP Award No. 0853498 and by the Swiss National Science Foundation. We acknowledge many useful discussions with A. Bovet, A. Fasoli, I. Furno, M. Gilmore, F. Halpern, J. Loizu, and C. Theiler.

${ }^{1}$ J. Ambrosiano, W. H. Matthaeus, M. L. Goldstein, and D. Plante, J. Geophys. Res. 93, 14383, doi: 10.1029/JA093iA12p14383 (1988).

${ }^{2}$ W. Heidbrink and G. Sadler, Nucl. Fusion 34, 535 (1994).

${ }^{3}$ ITER Physics Expert Group on Energetic Particles, Heating and Current Drive and ITER Physics Basis Editors, Nucl. Fusion 39, 2495 (1999).

${ }^{4}$ A. Fasoli, C. Gormenzano, H. L. Berk, B. Breizman, S. Briguglio, D. S. Darrow, N. Gorelenkov, W. W. Heidbrink, A. Jaun, S. V. Konovalov, R. Nazikian, J.-M. Noterdaeme, S. Sharapov, K. Shinohara, D. Testa, K. Tobita, Y. Todo, G. Vlad, and F. Zonca, Nucl. Fusion 47, 264 (2007).

${ }^{5}$ S. Günter, G. Conway, S. da Graça, H.-U. Fahrbach, C. Forest, M. G. Muñoz, T. Hauff, J. Hobirk, V. Igochine, F. Jenko, K. Lackner, P. Lauber, P. McCarthy, M. Maraschek, P. Martin, E. Poli, K. Sassenberg, E. Strumberger, G. Tardini, E. Wolfrum, H. Zohm, and A. U. Team, Nucl. Fusion 47, 920 (2007).

${ }^{6}$ T. Dannert, S. Günter, T. Hauff, F. Jenko, X. Lapillonne, and P. Lauber, Phys. Plasmas 15, 062508 (2008).

${ }^{7}$ W. W. Heidbrink, J. M. Park, M. Murakami, C. C. Petty, C. Holcomb, and M. A. V. Zeeland, Phys. Rev. Lett. 103, 175001 (2009).

${ }^{8}$ ITER Physics Expert Group on Energetic Particles, Heating and Current Drive and ITER Physics Basis Editors, Nucl. Fusion 39, 2471 (1999).

${ }^{9}$ M. Albergante, J. P. Graves, A. Fasoli, F. Jenko, and T. Dannert, Phys. Plasmas 16, 112301 (2009).

${ }^{10}$ K. Gustafson, P. Ricci, I. Furno, and A. Fasoli, Phys. Rev. Lett. 108, 035006 (2012)

${ }^{11}$ E. W. Montroll and G. H. Weiss, J. Math. Phys. 6, 167 (1965).

${ }^{12}$ R. Metzler and J. Klafter, Phys. Rep. 339, 1 (2000).

${ }^{13}$ Anomalous Transport: Foundations and Applications, edited by R. Klages, G. Radons, and I. Sokolov (Wiley-VCH, Weinheim, 2008).

${ }^{14}$ J. Klafter, A. Blumen, and M. Shlesinger, Phys. Rev. A 35, 3081 (1987)

${ }^{15}$ T. Solomon, E. R. Weeks, and H. L. Swinney, Phys. Rev. Lett. 71, 3975 (1993).

${ }^{16}$ M. A. Fogleman, M. J. Fawcett, and T. H. Solomon, Phys. Rev. E 63, 4 (2001).

${ }^{17}$ J. K. Lawrence, A. C. Cadavid, A. Ruzmaikin, and T. E. Berger, Phys. Rev. Lett. 86, 5894 (2001).

${ }^{18}$ G. Zimbardo, A. Greco, and P. Veltri, Phys. Plasmas 7, 1071 (2000).

${ }^{19}$ K. L. Wong, M. Ono, and G. A. Wurden, Rev. Sci. Instrum. 53, 409 (1982).

${ }^{20}$ E. D. Zimmerman and S. C. Luckhardt, J. Fusion Energy 12, 289 (1993).

${ }^{21}$ C. Riccardi, M. Fontanesi, A. Galassi, and E. Sindoni, Plasma Phys. Controlled Fusion 36, 1791 (1994)

${ }^{22}$ P. K. Sharma and D. Bora, Plasma Phys. Controlled Fusion 37, 1003 (1995).

${ }^{23}$ K. Rypdal and S. Ratynskaia, Phys. Rev. Lett. 94, 225002 (2005).

${ }^{24}$ K. W. Gentle and H. Huang, Plasma Sci. Technol. 10, 284 (2008). 
${ }^{25}$ P. Ricci, C. Theiler, A. Fasoli, I. Furno, B. Labit, S. H. Müller, M. Podestà, and F. M. Poli, Phys. Plasmas 16, 055703 (2009).

${ }^{26}$ P. Ricci, C. Theiler, A. Fasoli, I. Furno, K. Gustafson, D. Iraji, and J. Loizu, Phys. Plasmas 18, 032109 (2011).

${ }^{27}$ G. Plyushchev, A. Diallo, A. Fasoli, I. Furno, B. Labit, S. H. Müller, M. Podestà, F. M. Poli, H. Boehmer, W. W. Heidbrink, and Y. Zhang, Rev. Sci. Instrum. 77, 10F503 (2006).

${ }^{28}$ A. Bovet, A. Fasoli, I. Furno, K. Gustafson, and P. Ricci, "Investigation of fast ion transport in TORPEX," Nucl. Fusion (under review).

${ }^{29}$ A. Fasoli, B. Labit, M. McGrath, S. H. Müller, G. Plyushchev, M. Podestà, and F. M. Poli, Phys. Plasmas 13, 055902 (2006).

${ }^{30}$ A. Fasoli, A. Burckel, L. Federspiel, I. Furno, K. Gustafson, D. Iraji, B. Labit, J. Loizu, G. Plyushchev, P. Ricci, C. Theiler, A. Diallo, S. H. Mueller, M. Podestà, and F. Poli, Plasma Phys. Controlled Fusion 52, 124020 (2010).

${ }^{31}$ K. Gustafson, P. Ricci, A. Bovet, I. Furno, and A. Fasoli, "Suprathermal ion transport in simple magnetized torus configurations," Phys. Plasmas (under review).
${ }^{32}$ P. Ricci and B. N. Rogers, Phys. Plasmas 16, 062303 (2009).

${ }^{33}$ P. Ricci and B. N. Rogers, Phys. Rev. Lett. 104, 145001 (2010).

${ }^{34}$ F. M. Poli, P. Ricci, A. Fasoli, and M. Podestà, Phys. Plasmas 15, 032104 (2008).

${ }^{35}$ A. Zeiler, J. F. Drake, and B. Rogers, Phys. Plasmas 4, 2134 (1997).

${ }^{36}$ M. F. Shlesinger, J. Klafter, and Y. M. Wong, J. Stat. Phys. 27, 499 (1982).

${ }^{37}$ K. Gustafson, D. Del-Castillo-Negrete, and W. Dorland, Phys. Plasmas 15, 102309 (2008).

${ }^{38}$ G. S. Burillo, B. P. van Milligen, and A. Thyagaraja, Phys. Plasmas 16, 042319 (2009).

${ }^{39}$ G. Zumofen, A. Blumen, J. Klafter, and M. F. Shlesinger, J. Stat. Phys. 54, 1519 (1989).

${ }^{40}$ S. Zhou, W. W. Heidbrink, H. Boehmer, R. McWilliams, T. Carter, S. Vincena, S. K. P. Tripathi, P. Popovich, B. Friedman, and F. Jenko, Phys. Plasmas 17, 092103 (2010).

${ }^{41}$ S. Zhou, W. W. Heidbrink, H. Boehmer, R. McWilliams, T. A. Carter, S. Vincena, and S. K. P. Tripathi, Phys. Plasmas 18, 082104 (2011). 